\title{
A Decomposition and Dynamic Programming Aggregation Method for the Optimal Water Allocation of Reservoirs in Series
}

\section{Zhihao Gong}

Yangzhou University

Jilin Cheng ( $\nabla_{\text {jlcheng@yzu.edu.cn ) }}$

Yangzhou University https://orcid.org/0000-0002-8624-9223

Haomiao Cheng

Yangzhou University

Yi Gong

Yangzhou University

\section{Research Article}

Keywords: reservoirs in series, joint operation, multidimensional dynamic programming, decomposition, aggregation, meta-heuristic algorithm

Posted Date: June 3rd, 2021

DOI: https://doi.org/10.21203/rs.3.rs-431229/v1

License: (c) (i) This work is licensed under a Creative Commons Attribution 4.0 International License. Read Full License 


\title{
A Decomposition and Dynamic Programming Aggregation
}

\section{Method for the Optimal Water Allocation of Reservoirs in Series}

\author{
Zhihao Gong ${ }^{\mathrm{a}}$, Jilin Cheng ${ }^{\mathrm{a}^{*}}$, Haomiao Cheng ${ }^{\mathrm{b}}$, Yi Gong ${ }^{\mathrm{a}}$ \\ ${ }^{a}$ School of Hydraulic Science and Engineering, Yangzhou University, Yangzhou 225009, \\ China; ${ }^{b}$ School of Environmental Science and Engineering, Yangzhou University,
} Yangzhou 225127, China

*Corresponding author's email:jlcheng@yzu.edu.cn (Jilin Cheng)

\begin{abstract}
Research on water allocation of multiple reservoirs with the purpose of reducing water spills and improving the local runoff utilization is a matter of great concern in humid areas with uneven temporal and spatial distributions of water resources. An optimization model for a system of reservoirs in series is developed to minimize water shortages. Several constraints restrict the objective function, including available water, operation rules and water rights for replenishment of the reservoirs with water. The model features multiple dimensions with a single coupling constraint of the large-scale system. A decomposition and dynamic programming aggregation method (DDPA) is proposed; the subsystem models and the aggregation model are both solved with the classical one-dimensional dynamic programming. Compared with the conventional decomposition-coordination method, the proposed method is concise but reliable because it can directly use the results of subsystems to form the one-dimensional dynamic programming aggregation model, avoiding the iterative calculations according to the coordinating function. Compared with the meta-heuristic algorithms, the proposed method is more efficient because it is
\end{abstract}


independent of any algorithm parameter. The proposed method may provide a new reference for solving similar multi-reservoir optimization models.

\section{Keywords}

reservoirs in series, joint operation, multidimensional dynamic programming, decomposition, aggregation, meta-heuristic algorithm 


\section{Introduction}

Reservoirs are the main water sources in the hilly regions of southern China and

3 southeast Asia. The mean annual rainfall is over $1,000 \mathrm{~mm}$ in these humid regions, but

$470-80 \%$ of that occurs during the flood season due to the monsoon climate (Quinn et al.

5 2018). Although the annual total inflows into the local reservoirs are usually more than

6 what is needed to meet demands, a large amount of water is drained due to uneven

7 temporal and spatial distribution of inflows, which results in seasonal water shortages.

8 Because of this shortage, it is necessary to replenish a reservoir from other reservoirs

9 during the dry season (Ming et al. 2017; Reca et al. 2015), thus gradually forming a system

10 of reservoirs in series.

11 The basic principle of joint operation for a multi reservoir system is to redistribute

12 water resources through the hydraulic connections among connected reservoirs (Ahmad

13 et al. 2014). This setting can maximize the storage capacity of each reservoir and reduce

14 loss of water. Thus, the operation purpose of reservoirs in series is to reduce water spills

15 and improve runoff utilization; the decision variable is the amount of water supply in each

16 period, which is subject to the reservoir's capacities including water storage and water

17 supply (Rani et al. 2020; Chang et al. 2019; Birhanu et al. 2014; Sattari et al. 2009). The

18 number of decision variables and constraints increases with the growing number of

19 reservoirs in the system. Furthermore, hydraulic connections among the reservoirs should

20 be added into constraints, which inevitably complicate the model (Ehteram et al. 2017;

21 Ashrafi and Dariane 2017).

22 Mathematical programming methods are classical algorithms for solving optimal 
water allocation models of reservoirs. Dynamic programming based on Bellman's principle (Bellman and Dreyfus 1964) is highly applicable to this type of multi-stage decision-making process. However, the 'curse of dimensionality' is induced when dealing with a large number of reservoirs (Cheng et al. 2017; Chen et al. 2016). Decomposition (Turgeon 1981) is the mainstream idea to dealing with multidimensional optimization problems when using dynamic programming. The decomposition-coordination method (Mahey et al. 2017; Li et al. 2014; Tan et al. 2019) is most commonly used and it achieves the optimal state of the whole system through the successive iterative calculations between the large-scale system and subsystems according to the coordinating variables. Therefore, the convergence performance of the coordination variable has a great impact on the final results. In addition, the Lagrange multiplier method (Jafari and Alipoor 2011) is usually used to deal with the constraints when adopting the conventional decompositioncoordination method. However, it is restricted by the differentiability and convexity of the objective function and it may have difficulty in handling some complex constraints, for instance, the constraint which contains if statements.

Recently, meta-heuristic algorithms such as the genetic algorithm (Allawi et al. 2018a), the particle swarm optimization algorithm (Chen et al. 2018) and the ant colony algorithm (Moeini et al. 2013) have become the most popular methods for the optimization of reservoir operation models. The greatest advantage of meta-heuristic algorithms is that they can solve the multidimensional model without the decomposition operations. However, the globally optimal final results cannot be guaranteed because decision variables are randomly sampled and updated within the feasible regions 
according to the specific iteration rules, which is a common limitation among these algorithms (Hossain and El-shafie 2013; Allawi et al. 2018b). Furthermore, when the meta-heuristic algorithms are adopted, a constrained optimization problem usually transforms into an unconstrained one by penalty functions or other methods (Wan et al. 2016; Pina et al., 2017). Nevertheless, the meta-heuristic algorithms may fail to handle models with some complicated constraints. What is worse, the algorithm parameters, such as the crossover and mutation rates in GA and the inertia weight and acceleration coefficients in PSO, cannot be clearly determined despite the fact that these values are important factors which can affect the performances of algorithms (Zhang and Dong 2019).

There are only empirical value ranges for most algorithm parameters. Various approaches for setting parameters such as the deterministic strategy (Draa et al. 2015) and the adaptive strategy (Harrison et al. 2018; Cui et al. 2016) are derived but they are not proven to be of universal significance (Karafotias et al. 2015).

In light of the shortcomings of the aforementioned algorithms, a decomposition and dynamic programming aggregation method (DDPA) is proposed for the optimal water allocation of reservoirs in series. The method was applied to a system of dual reservoirs and dual pumping stations in Nanjing, China to verify its performance.

\section{Model and method}

\subsection{Description of the system}

A system of reservoirs in series is a common water supply system in hilly regions of southern China. There are several reservoirs with hydraulic connections between each other in the system as shown in Figure 1(a). The reservoirs usually operate jointly but have 

gravity.

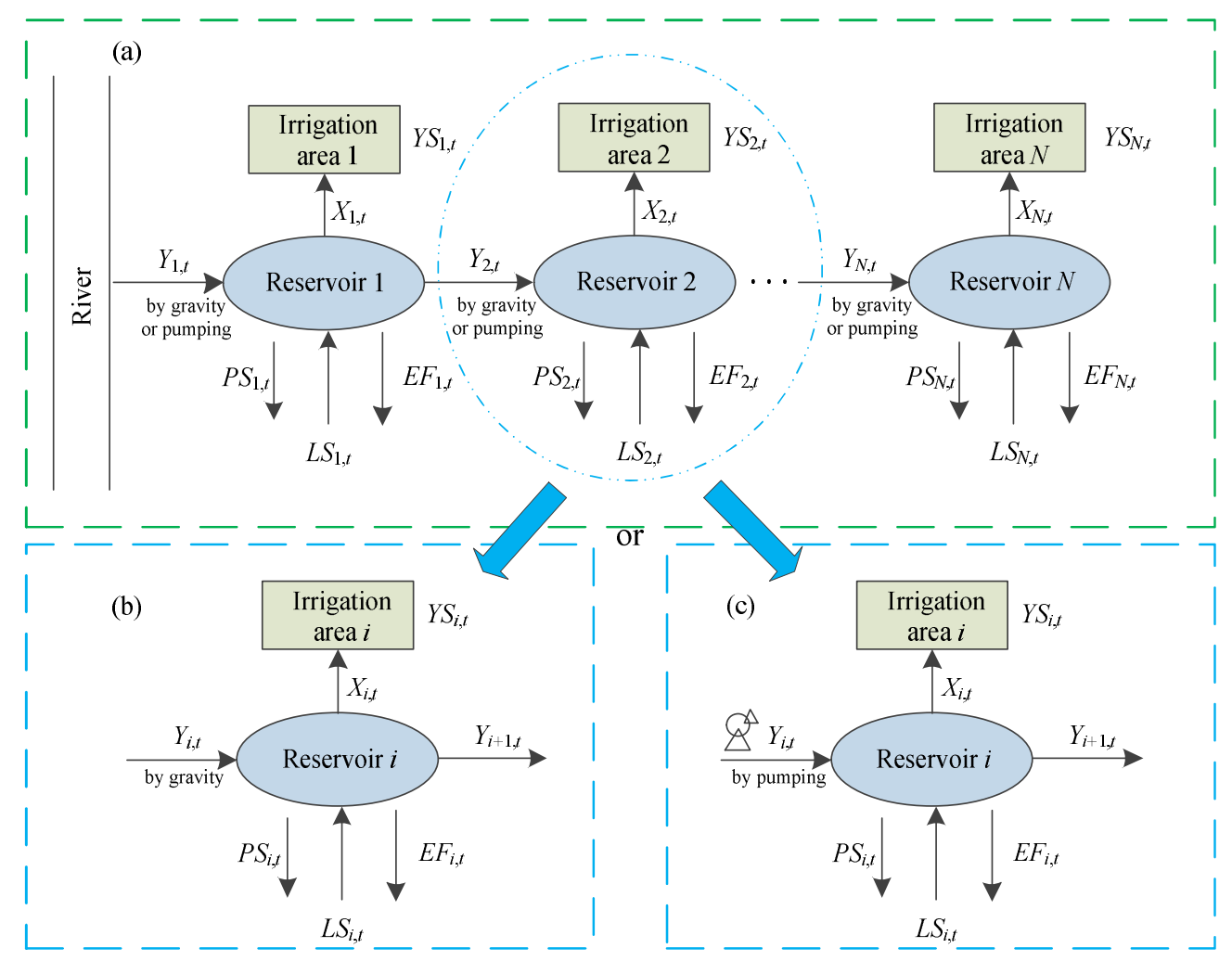

71 Note: (a) a system of reservoirs in series; (b) a subsystem of a reservoir; (c) a subsystem of a reservoir and a pumping station

In Figure 1: $X_{i, t}\left(\mathrm{~L}^{3}\right), Y S_{i, t}\left(\mathrm{~L}^{3}\right), L S_{i, t}\left(\mathrm{~L}^{3}\right), Y_{i, t}\left(\mathrm{~L}^{3}\right), P S_{i, t}\left(\mathrm{~L}^{3}\right)$ and $E F_{i, t}\left(\mathrm{~L}^{3}\right)$ are water supply, water demand, local inflow, water replenishment, water spill and evaporation of reservoir $i$ in period $t$, respectively, $N$ is the total number of reservoirs in the system, $i$ is the sequence number of reservoir, $i=1,2, \ldots, N$, and $t$ is the sequence number of each period. 
81 is to minimize the annual sum of the squared water shortage of each reservoir (Celeste and Billib 2009; Jothiprakash et al. 2011), which can be expressed as Equation (1):

$$
\min F=\sum_{i=1}^{N} \sum_{t=1}^{T}\left(X_{i, t}-Y S_{i, t}\right)^{2}
$$

where $F$ is the annual sum of squared water shortage of each reservoir in each period and $T$ is the total number of periods.

\subsubsection{Constraints}

(1) Annual available water of the whole system rights, which can be expressed as Equation (2):

$$
\sum_{i=1}^{N} \sum_{t=1}^{T} X_{i, t} \leq S K+B Z
$$

where $S K$ is the total annual available water of the reservoirs and $B Z$ is the maximum volume that can be acquired from outside water sources. replenishments from other water sources, which can be expressed as Equation (3):

$$
\sum_{t=1}^{T} X_{i, t}=W_{i}(i=1,2, \ldots, N)
$$
requirement of Equation (4): 
103 expressed as Equation (5):

104

$$
V_{i, t}^{\min } \leq V_{i, t} \leq V_{i, t}^{\max }
$$

105

106

107

108 where $V_{i, t}\left(\mathrm{~L}^{3}\right)$ is the water storage of reservoir $i$ during period $t$ and $V_{i, t}^{\min }\left(\mathrm{L}^{3}\right)$ and $V_{i, t}^{\max }$ $\left(\mathrm{L}^{3}\right)$ are the lower and upper bounds of storage during period $t$, respectively.

According to the water balance equation, the water storage of reservoir $i$ in period $t$ can be determined by Equation (6):

$$
V_{i, t}=V_{i, t-1}+L S_{i, t}-X_{i, t}-Y_{i+1, t}-E F_{i, t}
$$

111 the lower and upper bounds of water storage as follows:

112 a. If $V_{i, t}<V_{i, t}^{\min }$, then the reservoir should be replenished with water. $Y_{i, t}$ and $P S_{i, t}$ can be 113 determined by Equation (7) and Equation (8), respectively.

$$
Y_{i, t}=\min \left(V_{i, t}^{\min }-V_{i, t}, Y_{i, t}^{\max }\right)
$$

b. If $V_{i, t}>V_{i, t}^{\max }$, then excess water should be released. $Y_{i, t}$ and $P S_{i, t}$ can be determined by Equation (9) and Equation (10), respectively.

$$
P S_{i, t}=V_{i, t}-V_{i, t}^{\max }
$$

c. If $V_{i, t}^{\min } \leq V_{i, t} \leq V_{i, t}^{\max }$, then both of $Y_{i, t}$ and $P S_{i, t}$ should be zero, shown as Equation (11).

$$
Y_{i, t}=P S_{i, t}=0
$$

122 The correct water storage of each period can be obtained by Equation (12). 
(4) Maximum water supply can be expressed as Equation (13)

(5) Maximum water replenishment is restricted by the pumping capacity or water conveyance capability, which can be expressed as Equation (14):

$$
Y_{i, t} \leq Y_{i, t}^{\max }
$$

129 where $Y_{i, t}^{\max }\left(\mathrm{L}^{3}\right)$ is the maximum water replenishment of reservoir $i$ in period $t$.

A restriction was imposed to make the final storage of the reservoir equal to the initial

133 in the final period.

It is also necessary to introduce non-negativity constraints to avoid negative values,

136 which would be physically impossible.

\subsection{Solving methods}

In the above model, the constraints from $\square$ to $\square$ can be transformed into the feasible

139 regions of the decision variable $X_{i, t}$. Therefore, it is a dynamic programming problem of

$140 N+1$ dimensions, which is restricted by the constraints $\square$ and $\square$. A decomposition and

141 dynamic programming aggregation method for this optimal water allocation of reservoirs

142 in series is proposed in this paper, which solves both of the subsystem models and the

143 aggregation model with dynamic programming. In order to evaluate the solving 144 performance, two commonly used meta-heuristic algorithms, the genetic algorithm and 
145 particle swarm optimization algorithm, are adopted simultaneously.

146 1.3.1 Decomposition and dynamic programming aggregation

147 (1) Decomposition of the system

148 The 'curse of dimensionality' will inevitably arise if using dynamic programming

149 (DP) to solve the model directly, hence it is necessary to first reduce the dimensions. The

150 large-scale system will then be decomposed into several subsystems and each subsystem

151 only consists of a reservoir or a reservoir and a pumping station as shown in Figure 1(b)

152 and (c).

153 (2) Optimization of subsystems

154 The optimization model of the subsystem is expressed as Equation (15) and (16),

155 where the objective function is expressed as

$$
\min f_{i}=\sum_{t=1}^{T}\left(X_{i, t}-Y S_{i, t}\right)^{2}
$$

157 and the annual available water of subsystem is expressed as

$$
\sum_{i=1}^{T} X_{i, t}=W_{i}
$$

159 where $f_{i}$ is the annual sum of squared water shortage in each period of subsystem $i$.

160 Moreover, the lower and upper bounds of water storage, water balance equation,

161 initial and boundary conditions should also be imposed on each subsystem, which can be

162 considered in the operation rule. The operation rule is integrated into recursive procedures

163 of DP to correct water storage $V_{i, t}$ and obtain water spill $P S_{i, t}$ and water replenishment $Y_{i, t}$

164 of each period simultaneously. In this manner, one-dimensional dynamic programming

165 can be adopted to solve the subsystem models as shown in Figure 2(a). 
For subsystem $i$, the annual available water $W_{i}$ can be discretized in its feasible region,

168 and the objective function value $f_{i}$ can be obtained under each $W_{i}$. After that, the

169 aggregation model can be formed directly using the optimization results of subsystems

$170 f_{i}\left(W_{i}\right)$, which can be expressed as Equation (18) and (19).

171

Objective function:

172

$$
\min F=\sum_{i=1}^{N} \sum_{t=1}^{T}\left(X_{i, t}-Y S_{i, t}\right)^{2}=\sum_{i=1}^{N} f_{i}\left(W_{i}\right)
$$

173

Coupling constraint:

$$
\sum_{i=1}^{N} \sum_{t=1}^{T} X_{i, t}=\sum_{i=1}^{N} W_{i} \leq S K+B Z
$$

The aggregation model is also a typical one-dimensional dynamic programming model, where the decision variable is the annual available water of subsystem $W_{i}$. The

177 inverse recursion of DP should be applied as shown in Figure 2(b), so that the water

178 replenishment from subsystem $i-1$ to subsystem $i$ can be known when the model of

179 subsystem $i-1$ is optimized. The state transition equation can be expressed as Equation (20)

$$
S W_{i}=S W_{i-1}-W_{i-1}(i=2,3, \ldots, N)
$$

181 where $S W_{i}\left(\mathrm{~L}^{3}\right)$ is the sum of available water in subsystems from $i$ to $N, S W_{i-1}\left(\mathrm{~L}^{3}\right)$ is the

182 sum of available water in subsystems from $i-1$ to $N$, and $W_{i-1}\left(\mathrm{~L}^{3}\right)$ is the available water 183 in subsystem $i-1$. 


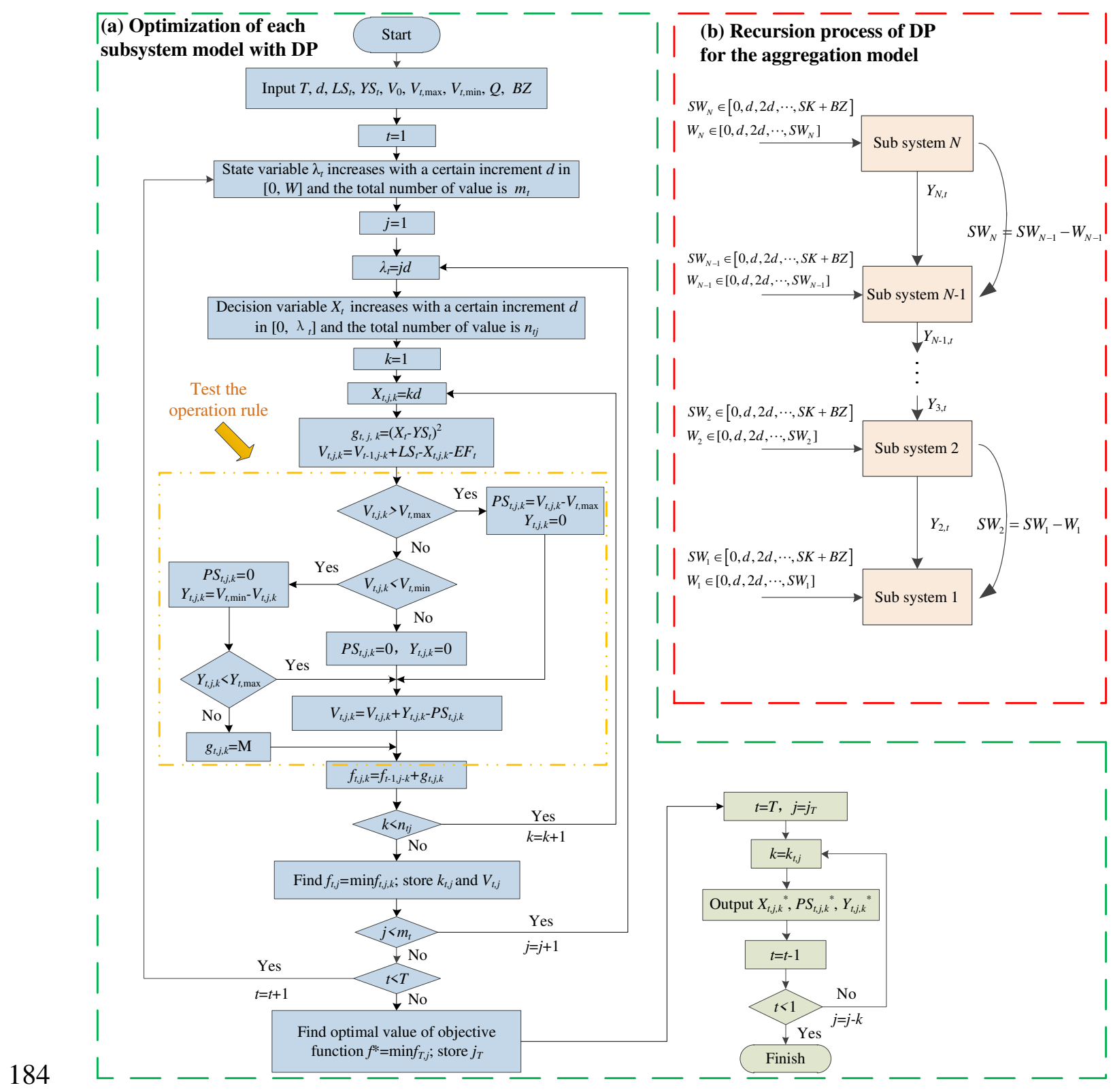

185 Note: $d$ is a certain increment; the aggregation model is optimized in reverse order so that

$186 Y_{i+1}$ is a known quantity when $V_{i}$ is calculated according to Equation (6); $\mathrm{M}$ is a positive

187 number, which is large enough.

189 After obtaining the optimal results of the aggregation model $F^{*}$ and $\left[W_{1}{ }^{*}, W_{2}{ }^{*}, \ldots\right.$,

$\left.190 W_{N}^{*}\right]$, the optimization results $\left[X_{i, t}, P S_{i, t}, Y_{i, t}\right]^{*}$ of each subsystem can be acquired by

191 searching the results of the subsystem models according to $W_{i}^{*}$, which can finally compose 
192

an optimal operation scheme of the whole system.

In conclusion, the essence of the decomposition and dynamic programming aggregation method is to transform the $N+1$ dimensional dynamic programming into the $N+1$ iterative calculations of one-dimensional dynamic programming. In this process, both of the subsystem models and the aggregation model are one-dimensional dynamic programming models. Therefore, the 'curse of dimensionality' can be avoided and the global optimal results can be acquired based on Bellman's principle.

\subsubsection{Genetic algorithm and particle swarm optimization algorithm}

(1) Genetic algorithm

The genetic algorithm (GA) has been successfully applied to the different kinds of optimal reservoir operation problems (Zhu et al. 2014; Tsai et al. 2019). The optimizing procedure of GA is based on the natural selection: initial chromosomes (solutions) are randomly generated first and the next generations are updated through the selection, crossover and mutation operators until the termination criterion is met. In each iteration, the selection operator is carried out according to the principle of roulette. Equation (21) and Equation (22) are used for the crossover and mutation operators, respectively,

$$
\begin{gathered}
X_{i, t}^{\text {child }}=\alpha X_{i, t}^{\mathrm{par} 1}+(1-\alpha) X_{i, t}^{\mathrm{par} 2} \\
X_{i, t}^{\text {new }}=\beta Y S_{i, t}
\end{gathered}
$$

where $X_{i, t}^{\text {child }}$ is an individual offspring generated through the crossover operator, $X_{i, t}^{\text {par1 }}$ and $X_{i, t}^{\text {par2 }}$ are both parent individuals, $X_{i, t}^{\text {new }}$ is a new individual generated through the mutation operator, and $\alpha$ and $\beta$ are both random values that vary in $[0,1]$.

(2) Particle swarm optimization algorithm 
216 The optimizing procedure of PSO starts with a number of initial particles provided

217 stochastically in the feasible region. Each particle's position vector and velocity vector 218 are updated according to Equations (23) and (24) in each iteration until the termination 219 criterion is met:

$$
v_{s}(k)=\omega v_{s}(k-1)+c_{1} r_{1}\left(p_{s}(k-1)-\theta_{s}(k-1)\right)+c_{2} r_{2}\left(g(k-1)-\theta_{s}(k-1)\right)
$$

$$
\theta_{s}(k)=\theta_{s}(k-1)+v_{s}(k)
$$

222 where $v_{s}$ is the velocity vector of particle $s, \theta_{s}$ is the position vector of particle $s, k$ is the 223 number of iteration times, $\omega$ is the inertia weight, $p_{s}$ is the best position of particle $s, g$ is 224 the global best solution among all the particles, $c_{1}$ and $c_{2}$ are acceleration coefficients and $225 \quad r_{1}$ and $r_{2}$ are random values that vary in $[0,1]$

\section{Case study}

In order to compare the performances of the three algorithms, the model is applied to a dual-reservoir-and-dual-pumping-station system in Nanjing, China, which consists of 229 the SH reservoir, the HWB reservoir and two pumping stations, HZ and XZ, as shown in

230 Figure 3 . The system is located at $32^{\circ} 27^{\prime} \mathrm{N}, 118^{\circ} 46^{\prime} \mathrm{E}$, in the subtropical monsoon 231 climate zone. The mean annual rainfall is $1002.7 \mathrm{~mm}$ in this region but $73.6 \%$ of the 232 annual rainfall occurs between June and September during the flood season.

The system provides irrigation water and each reservoir provides water

234 independently to its own irrigation area. The average topography of the HWB reservoir is 
236 can replenish the HWB reservoir with water from the SH reservoir, while the SH reservoir

237 can be replenished with water from the BL river through the XZ pumping station.

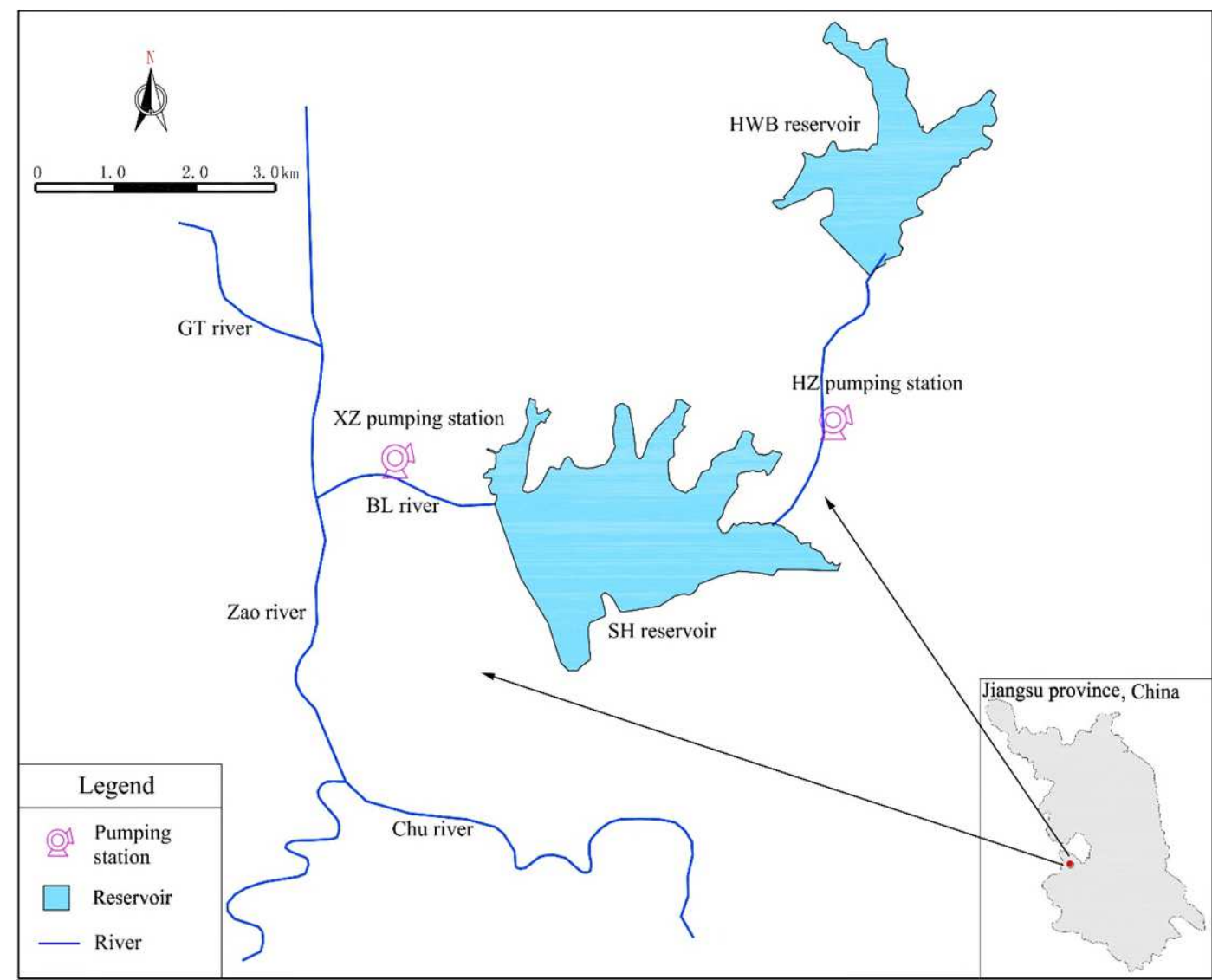

Figure 3 Location of the system

(1) Reservoir and pumping station

The characteristics of reservoirs and pumping stations are shown in Tables 1 and 2,

242 respectively.

Table 1 Characteristics of reservoirs

\begin{tabular}{llllll}
\hline & Dead storage & Utilizable & Total storage & Limited storage \\
Reservoir & capacity $\left(10^{4}\right.$ & $\begin{array}{l}\text { capacity }\left(10^{4}\right. \\
\text { capacity } \\
\left.\mathrm{m}^{3}\right)\end{array}$ & 1157 & $\begin{array}{l}\text { capacity in flood } \\
\left(10^{4} \mathrm{~m}^{3}\right)\end{array}$ & $\begin{array}{l}\text { Irrigation area } \\
\left(\mathrm{hm}^{2}\right)\end{array}$ \\
\hline SH & 600 & 2473 & 1000 & 1667 \\
HWB & 457 & 936 & 1891 & 1393 & 2067 \\
\hline
\end{tabular}




\begin{tabular}{lllll}
\hline Pumping station & $\begin{array}{l}\text { Design discharge } \\
\left(\mathrm{m}^{3} / \mathrm{h}\right)\end{array}$ & $\begin{array}{l}\text { Design pumping } \\
\text { head }(\mathrm{m})\end{array}$ & $\begin{array}{l}\text { Maximum daily } \\
\text { operation } \\
\text { duration }(\mathrm{h})\end{array}$ & $\begin{array}{l}\text { Water rights (75\%) } \\
\left(10^{4} \mathrm{~m}^{3}\right)\end{array}$ \\
\hline $\mathrm{XZ}$ & 10000 & 19.4 & 20 & 360 \\
$\mathrm{HZ}$ & 7500 & 15.0 & 20 & $/$ \\
\hline
\end{tabular}

246 Notes: The annual water rights of the $\mathrm{XZ}$ pumping station, which means the maximum

247 volume that can be pumped from the BL river, are allocated by the local water authority;

248 the HZ pumping station is an internal pumping station in the system and is not defined or

249 limited by any water rights.

250 (2) Inflows and water demands

251 The inflow and water demand in each month at $75 \%$ probability of exceedance are

252 shown in Table 3.

Table 3 Monthly inflows and water demands unit: $10^{4} \mathrm{~m}^{3}$

\begin{tabular}{llccccccccccccc}
\hline \multirow{2}{*}{ Reservoir } & \multirow{2}{*}{ Category } & \multicolumn{10}{c}{ Period } & \multicolumn{10}{c}{ Total } \\
\cline { 3 - 12 } & & Oct. & Nov. & Dec. & Jan. & Feb. & Mar. & Apr. & May & Jun. & Jul. & Aug. & Sep. \\
\hline \multirow{2}{*}{ SH } & Inflows & 115 & 45 & 11 & 32 & 2 & 28 & 32 & 50 & 445 & 781 & 618 & 320 & 2479 \\
& Demands & 23 & 15 & 18 & 17 & 23 & 26 & 30 & 31 & 523 & 323 & 159 & 33 & 1221 \\
\hline \multirow{2}{*}{ HWB } & Inflows & 33 & 55 & 1 & 36 & 10 & 9 & 37 & 56 & 218 & 133 & 157 & 20 & 766 \\
& Demands & 59 & 79 & 17 & 16 & 23 & 27 & 31 & 35 & 550 & 559 & 345 & 77 & 1817 \\
\hline
\end{tabular}

(3) Evaporation

256 a reservoir in each period, which can be described as Equation (25). The average free

257 water surface of each reservoir in a specific period is determined by its surface-volume

258 relationship, which was provided by Liuhe Water Authority.

$$
E F_{t}=0.1 \times k_{t} \times E_{t} \times\left(\alpha V_{t}+\beta\right)
$$

260 where $E F_{t}\left(10^{4} \mathrm{~m}^{3}\right)$ is the evaporation loss of a reservoir in period $t, E_{t}(\mathrm{~mm})$ is the 
261 evaporation depth of $E_{601}$ evaporator in period $t$ as shown in Table $4, k_{t}$ is the correction

262 coefficient for period $t$ as shown in Table $4, V_{t}\left(10^{4} \mathrm{~m}^{3}\right)$ is the average water storage in 263 period $t$ and $\alpha$ and $\beta$ are the reservoir coefficients. For the SH reservoir, $\alpha=1.194 \times 10^{-3}, \beta$

$264=2.575$, and for the HWB reservoir, $\alpha=1.657 \times 10^{-3}, \beta=1.862$.

Table $4 E_{t}$ and $k_{t}$ of each month

\begin{tabular}{lcccccccccccc}
\hline Period & Oct. & Nov. & Dec. & Jan. & Feb. & Mar. & Apr. & May & Jun. & Jul. & Aug. & Sep. \\
\hline$E_{t}(\mathrm{~mm})$ & 56 & 42 & 38 & 23 & 28 & 41 & 58 & 86 & 98 & 115 & 110 & 71 \\
$k_{t}$ & 1.04 & 1.12 & 1.12 & 1.05 & 0.92 & 0.9 & 0.88 & 0.92 & 0.94 & 0.94 & 0.98 & 1.06 \\
\hline
\end{tabular}

2663 Results and analysis

\subsection{Optimization Results}

The results obtained with different algorithms are shown in Table 5. The conventional operation of the system was conducted in Excel-2010 according to the standard operation

270 policy (SOP), while the other three optimization methods were programmed in Visual

271 Basic language based on a PC with i3 CPU/3.70 GHz/4 GB ram.

272 Compared with the results using SOP, the water allocation of the system can be

273 optimized by using the optimization methods. By increasing the water diverted from the

274 SH reservoir to the HWB reservoir, the total water spill of the SH reservoir and the total

275 water shortage of the HWB reservoir were reduced. Among the three optimization 276 methods, DDPA minimized the objective function better than the other algorithms. The 277 value of objective function obtained with DDPA was decreased by $7.5 \%$ and $5.6 \%$ when 278 compared to GA and PSO, respectively.

279 Although the amount of water replenishment from the BL river was same, the total 280 water spill of the system obtained with DDPA was reduced by $2.0 \%$ while the total water 281 shortage was reduced by $5.3 \%$ and $10.0 \%$ when compared to GA and PSO, respectively. 
282 It is indicated the utilization rate of local runoff in the DDPA results is relatively higher 283 on premise of the same external water rights.

284 On the other hand, the operating time of DDPA was 3.85 times and 3.10 times than 285 GA and PSO respectively, though the results obtained with DDPA were better. As shown 286 in Table 5, GA and PSO are more efficient in each operation when the algorithm 287 parameters are determined. However, the operating time is quite longer when considering 288 the tests of the appropriate algorithm parameters of GA and PSO, which is discussed in 289 the next section.

\begin{tabular}{|c|c|c|c|c|c|c|c|c|c|}
\hline Methods & Reservoir & $\begin{array}{l}\text { Water } \\
\text { supply }\end{array}$ & $\begin{array}{l}\text { Water } \\
\text { spill }\end{array}$ & $\begin{array}{c}\text { Water } \\
\text { replenishment }\end{array}$ & $\begin{array}{c}\text { Water } \\
\text { shortage }\end{array}$ & Evaporation & $\begin{array}{l}\text { Objective } \\
\text { function }\end{array}$ & $\begin{array}{c}\text { Single } \\
\text { operation } \\
\text { time } \\
\text { (s) }\end{array}$ & $\begin{array}{c}\text { Total } \\
\text { operation } \\
\text { time } \\
\text { (s) }\end{array}$ \\
\hline \multirow{3}{*}{ SOP } & $\mathrm{SH}$ & 1221 & 241 & 360 & 0 & 278 & \multirow{3}{*}{ I } & \multirow{3}{*}{ I } & \multirow{3}{*}{ I } \\
\hline & HWB & 1663 & 0 & 1099 & 154 & 202 & & & \\
\hline & Total & 2884 & 241 & 1459 & 154 & 480 & & & \\
\hline \multirow{3}{*}{ DDPA } & SH & 1221 & 98 & 356 & 0 & 272 & \multirow{3}{*}{234} & \multirow{3}{*}{24.05} & \multirow{3}{*}{24.05} \\
\hline & HWB & 1799 & 0 & 1243 & 18 & 210 & & & \\
\hline & Total & 3020 & 98 & 1599 & 18 & 482 & & & \\
\hline \multirow{3}{*}{ GA } & SH & 1221 & 100 & 356 & 0 & 272 & \multirow{3}{*}{251} & \multirow{3}{*}{6.24} & \multirow{3}{*}{1682} \\
\hline & HWB & 1798 & 0 & 1241 & 19 & 209 & & & \\
\hline & Total & 3019 & 100 & 1597 & 19 & 481 & & & \\
\hline \multirow{3}{*}{ PSO } & $\mathrm{SH}$ & 1221 & 100 & 356 & 0 & 272 & \multirow{3}{*}{248} & \multirow{3}{*}{7.77} & \multirow{3}{*}{1533} \\
\hline & HWB & 1797 & 0 & 1241 & 20 & 210 & & & \\
\hline & Total & 3018 & 100 & 1597 & 20 & 482 & & & \\
\hline
\end{tabular}

291 Notes: (1) The conventional operation was conducted according to the SOP regardless of

292 the objective function value and operation time; (2) the objective function value of GA is

293 the minimum value among five runs when the crossover rate, mutation rate and population

294 are 0.7, 0.03 and 200, respectively; (3) the objective function value of PSO is the minimum

295 value among five runs when the inertia weight, acceleration coefficients and population 
are 0.8, 2.1 and 200, respectively; (4) the single operation time is an average value when

297 the algorithm parameters are determined; (5) the total operation time contains the time 298 consumed for testing the appropriate algorithm parameters of GA and PSO.

299

300

301

302

\subsection{Evaluation indexes}

The volumetric reliability and vulnerability indexes (Chanda et al. 2014) were selected to evaluate the performances of different algorithms. The volumetric reliability index is expressed as Equation (26), which indicates the ratio of water supply to water demands. A high percentage of this index is desirable (Asefa et al. 2014). The vulnerability index is expressed as Equation (27), which represents the maximum failure rate during the operation. A low percentage of this index is desirable (Asefa et al. 2014).

$$
\begin{gathered}
\operatorname{Re} l_{i}=\frac{\sum_{t=1}^{T}\left(X_{i, t} / Y S_{i, t}\right)}{T} \\
V u l_{i}=\max _{t=1}^{T}\left\{1-\frac{X_{i, t}}{Y S_{i, t}}\right\}
\end{gathered}
$$

where $\operatorname{Rel}_{i}(\%)$ is the volumetric reliability index of reservoir $i$ and $\operatorname{Vul}_{i}(\%)$ is the vulnerability index of reservoir $i$.

The volumetric reliability and vulnerability indexes of different methods are shown in Table 6. For the SH reservoir, the water demand can be met entirely using the SOP or optimization methods because the available water in the reservoir is much more than the water demand. For the HWB reservoir, the volumetric reliability index of DDPA is $99.51 \%$, which is $1.5 \%, 0.02 \%$ and $0.05 \%$ more than that of SOP, GA and PSO, respectively. The vulnerability indexes of DDPA and GA are both $8.20 \%$, which are $20.93 \%$ and $0.54 \%$ less than that of SOP and PSO, respectively. Thus, the performance of DDPA is relatively 
317 better because it can simultaneously achieve a high percentage of volumetric reliability 318 index and a low percentage of vulnerability index.

Table 6 Volumetric reliability \& vulnerability

\begin{tabular}{cccc}
\hline Reservoir & Methods & Volumetric reliability $(\%)$ & Vulnerability $(\%)$ \\
\hline \multirow{3}{*}{ SH } & SOP & 100 & 0 \\
& DDPA & 100 & 0 \\
& GA & 100 & 0 \\
& PSO & 100 & 0 \\
\hline \multirow{3}{*}{ HWB } & SOP & 98.01 & 29.13 \\
& DDPA & 99.51 & 8.20 \\
& GA & 99.49 & 8.20 \\
\hline
\end{tabular}

4 Comparison and discussion

321 The random parameters of different meta-heuristic algorithms have a significant

322 influence on the performances of the algorithms. Objective function values obtained with

323 different algorithms under different combinations of parameters are shown in Figure 4.

324 The crossover rate and mutation rate of GA vary from 0.5 to 0.9 and 0.01 to 0.1 , 325 respectively, while the inertia weight and acceleration coefficients $\left(c_{1}=c_{2}\right)$ of PSO vary 326 from 0.5 to 0.9 and 1.1 to 2.5 , respectively. The population sizes of the two algorithms are 327 both 200.

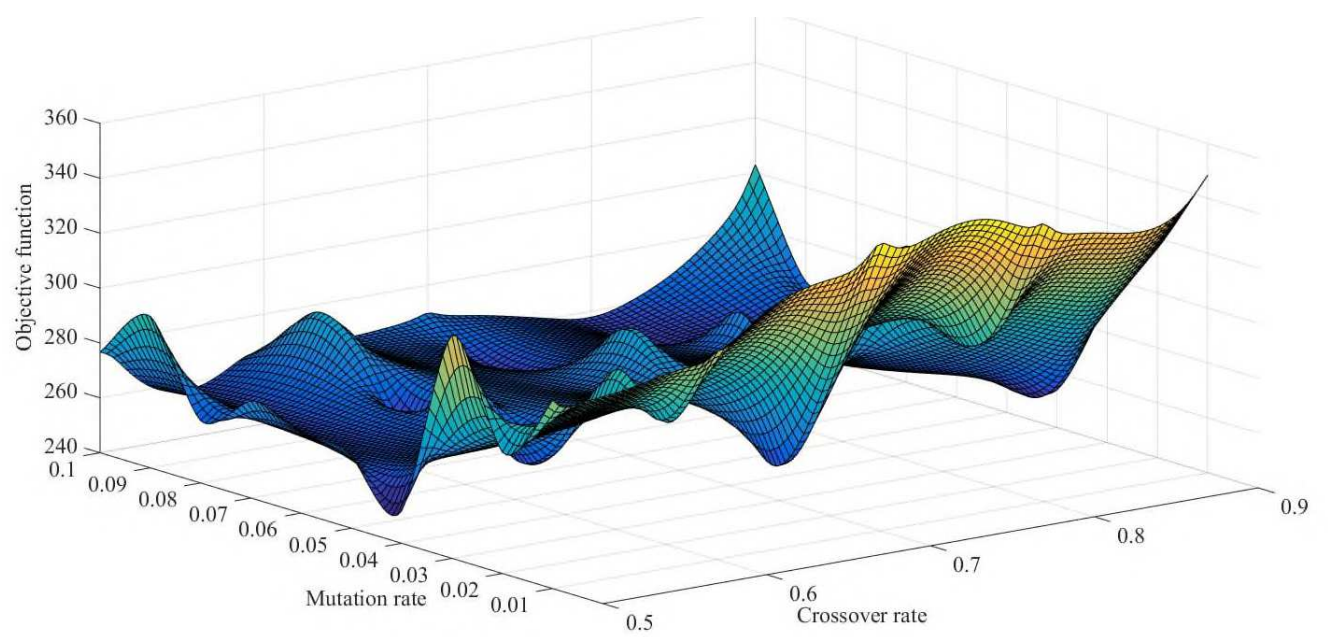

(a) Genetic algorithm 


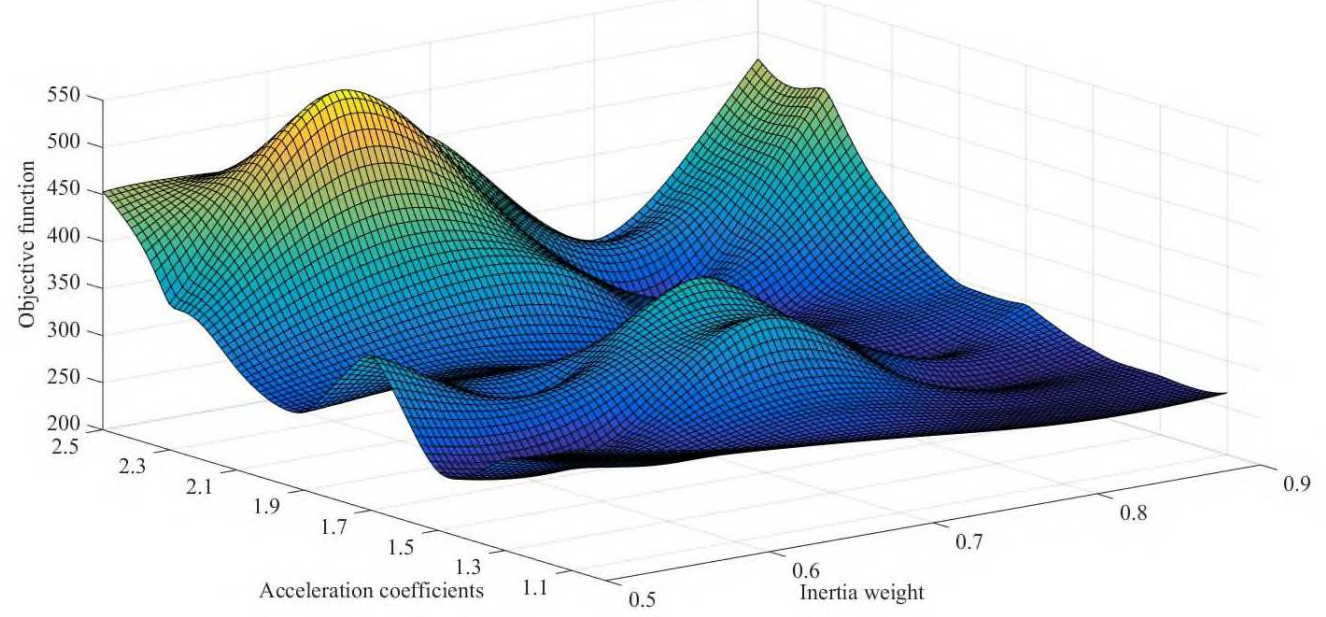

(b) Particle swarm optimization algorithm

332 Notes: Each objective function value under different combinations of parameters is the

333 minimum value among five runs.

Figure 4 The objective function values obtained with GA and PSO

As shown in Figure 4, the most appropriate crossover rate and mutation rate of GA are 0.7 and 0.03 , respectively, and the corresponding optimum objective function value is respectively, and the corresponding optimum objective function value is 248 . The optimum objective function value obtained with DDPA is 234 and the difference among the three algorithms is minor, which can verify the reliabilities of each other.

342 parameters, which can be quite different depending on various factors. On the contrary,

343 there is no additional parameter in DDPA and the dynamic programming based on 344 Bellman's principle can guarantee the global optimal results. Therefore, the final optimal 345 results achieved by DDPA are always the same while the actual operation time just varies 346 within a minor range. Correspondingly, if considering the time consumed for testing the 
347 algorithm parameters, the operation time of GA or PSO would be much longer than that 348 of DDPA as shown in Table 5.

\section{Conclusion}

This study developed an optimization model for the system of reservoirs in series to

351 minimize water shortage, which is restricted by available water, operation rules and water 352 rights for replenishment with water. A decomposition and dynamic programming 353 aggregation method was proposed to resolve this model. The primary characteristic of the 354 method is that the subsystem models and the aggregation model are both solved with the 355 classical one-dimensional dynamic programming. Therefore, it can reduce the dimension 356 by transforming the $N+1$ dimensional dynamic programming into the $N+1$ iterative 357 calculations of one-dimensional dynamic programming.

358 Compared with the conventional decomposition-coordination method, the proposed 359 method is concise but reliable because it can directly use the results of subsystems to form 360 the one-dimensional dynamic programming aggregation model, avoiding the iterative 361 calculations according to the coordinating function. Compared with the meta-heuristic 362 algorithms, the proposed method is more efficient because it is independent of any 363 algorithm parameter.

\section{Declarations}

365 Acknowledgements: the authors wish to acknowledge the managers of Liuhe Water 366 Authority, Nanjing, China, for their support of providing reservoir, irrigation and 367 hydrology data.

368 Funding: this work was supported by the National Natural Science Foundation of China 
369 (NSFC) [grant number 52079119] and National Key Research and Development Program

370 of China [grant number 2017YFC0403205].

371 Conflicts of interest/Competing interests: none.

372 Availability of data and material (data transparency): all of data can be found in this

373 paper.

374 Code availability: the codes for DDPA, GA and PSO and detailed solving results are

375 available at https://github.com/ChengJilin/Codes-in-manuscript-for-WRM-.git.

376 Ethics approval: not applicable.

377 Consent to participate: not applicable.

378 Consent for publication: not applicable.

379 References

380 Ahmad A, El-Shafie A, Razali SFM, Mohamad ZS, (2014) Reservoir optimization in 381 water resources: a review. Water Resources Management 28: 3391-3405.

$382{ }^{a}$ Allawi MF, Jaafar O, Ehteram M, Hamzah FM, El-Shafie A, (2018) Synchronizing 383 artificial intelligence models for operating the dam and reservoir system. Water $384 \quad$ Resources Management 32: 3373-3389.

$385{ }^{b}$ Allawi MF, Jaafar O, Mohamad HF, Abdullah SMS, El-shafie A (2018) Review on 386 applications of artificial intelligence methods for dam and reservoir-hydro387 environment models. Environmental Science \& Pollution Research 25: 13446-13469. 388 Asefa Y, Clayton J, Adams A, Anderson D (2014) Performance evaluation of a water 389 resources system under varying climatic conditions: reliability, resilience, 390 vulnerability and beyond. Journal of Hydrology 508: 53-56. 
Ashrafi SM, Dariane AB (2017) Coupled operating rules for optimal operation of multireservoir systems. Water Resources Management 31: 4505-4520.

Bellman RE, Dreyfus SE (1964) Applied dynamic programming. Princeton University Press, London.

Birhanu K, Alamirew T, Dinka MO, Ayalew S, Aklog D (2014) Optimizing reservoir operation policy using chance constraint nonlinear programming for Koga irrigation dam, Ethiopia. Water Resources Management 28: 4957-4970.

Celeste A B, Billib M (2009) The role of spill and evaporation in reservoir optimization models. Water Resources Management 24: 617-628.

Chanda K, Maity R, Sharma A, Mehrotra R (2014) Spatiotemporal variation of long-term drought propensity through reliability-resilience-vulnerability based drought management index. Water Resources Research 50: 7662-7676.

Chang J, Guo A, Wang Y, Ha Y, Zhang R, Xue L, Tu Z (2019) Reservoir operations to mitigate drought effects with a hedging policy triggered by the drought prevention limiting water level. Water Resources Research 55: 904-922.

Chen D, Leon AS, Gibson NL, Hosseini P (2016) Dimension reduction of decision variables for multireservoir operation: a spectral optimization model. Water Resources Research 52: 36-51.

Chen S, Lei C, Little JC, Carey CC, McClure RP, Lofton ME (2018) Three-dimensional effects of artificial mixing in a shallow drinking-water reservoir. Water Resources Research 54: 425-441.

Cheng C, Wang S, Chau K, Wu X (2017) Parallel discrete differential dynamic 

57: $152-164$.

Cui L, Li G, Lin Q, Chen J, Lu N (2016) Adaptive differential evolution algorithm with novel mutation strategies in multiple sub-populations. Computers \& Operations Research 64: 155-173.

Draa A, Bouzoubia S, Boukhalfa I (2015) A sinusoidal differential evolution algorithm for numerical optimization. Applied Soft Computing 27: 99-126.

Ehteram M, Mousavi SF, Karami H, Farzin S, Emami M, Othman FB, Amini Z, Kisi O, 287-298.

Harrison KR, Engelbrecht AP, Ombuki-Berman BM (2018) Self-adaptive particle swarm optimization: a review and analysis of convergence. Swarm Intelligence 12: 187-226.

Hossain MS, El-shafie A (2013) Intelligent systems in optimizing reservoir operation

Jafari H, Alipoor A (2011) A new method for calculating general Lagrange multiplier in the variational iteration method. Numerical Methods for Partial Differential

Jothiprakash V, Shanthi G, Arunkumar R (2011) Development of operational policy for a multi-reservoir system in India using genetic algorithm. Water Resources Management 25: 2405-2423.

434 Karafotias G, Hoogendoorn M, Eiben AE (2015) Parameter control in evolutionary 

19: 167-187.

Li C, Zhou J, Ouyang S, Ding X, Chen L (2014) Improved decomposition-coordination and discrete differential dynamic programming for optimization of large-scale hydropower system. Energy Conversion and Management 84: 363-373.

Mahey P, Koko J, Lenoir A (2017) Decomposition methods for a spatial model for longterm energy pricing problem. Mathematical Methods of Operations Research 85:

Ming B, Liu P, Chang J, Wang Y, Huang Q (2017) Deriving operating rules of pumped Management 143: 05017012.

Moeini R, Afshar MH (2013) Extension of the constrained ant colony optimization algorithms for the optimal operation of multi-reservoir systems. Journal of

Pina J, Tilmant A, Cote P (2017) Optimizing multireservoir system operating policies using exogenous hydrologic variables. Water Resources Research 53: 9845-9859.

Quinn JD, Reed PM, Giuliani M, Castelletti A, Oyler JW, Nicholas RE (2018) Exploring how changing monsoonal dynamics and human pressures challenge multireservoir management for flood protection, hydropower production, and agricultural water supply. Water Resources Research 54: 4638-4662.

456 Rani D, Mourato S, Moreira M (2020) A generalized dynamic programming modelling 
Reca J, García-Manzano A, Martíneza J (2015) Optimal pumping scheduling model considering reservoir evaporation. Agricultural Water Management 148: 250-257.

461 Sattari MT, Apaydin H, Ozturk F (2009) Operation analysis of Eleviyan irrigation reservoir dam by optimization and stochastic simulation. Stochastic Environmental Research and Risk Assessment 23: 1187-1201.

Tan Y, Dong Z, Xiong C, Zhong Z, Hou L (2019) An optimal allocation model for large complex water resources system considering water supply and ecological needs. Water 11: 843 .

Tsai W, Cheng C, Uen T, Zhou Y, Chang F (2019) Drought mitigation under urbanization through an intelligent water allocation system. Agricultural Water Management 213:

Turgeon A (1981) A decomposition method for the long-term scheduling of reservoir in

Wan F, Yuan W, Zhou J (2016) Derivation of tri-level programming model for multireservoir optimal operation in inter-basin transfer-diversion-supply project. Water Resources Management 31: 479-494.

Wan W, Guo X, Lei X, Jiang Y, Wang H (2018) A novel optimization method for multireservoir operation policy derivation in complex inter-basin water transfer system. Water Resources Management 32: 31-51.

478 Yousefi M, Banihabib ME, Soltani J, Roozbahani A (2018) Multi-objective particle swarm 

optimization model for conjunctive use of treated wastewater and groundwater. Agricultural Water Management 208: 224-231.

481 Zhang J, Dong Z (2019) Parameter combination framework for the differential evolution algorithm. Algorithms 12: 71.

483 Zhu X, Zhang C, Yin J, Zhou H, Jiang Y (2014) Optimization of water diversion based on 484 reservoir operating rules: analysis of the Biliu river reservoir, China. Journal of Hydrologic Engineering 19: 411-421. 
Figures

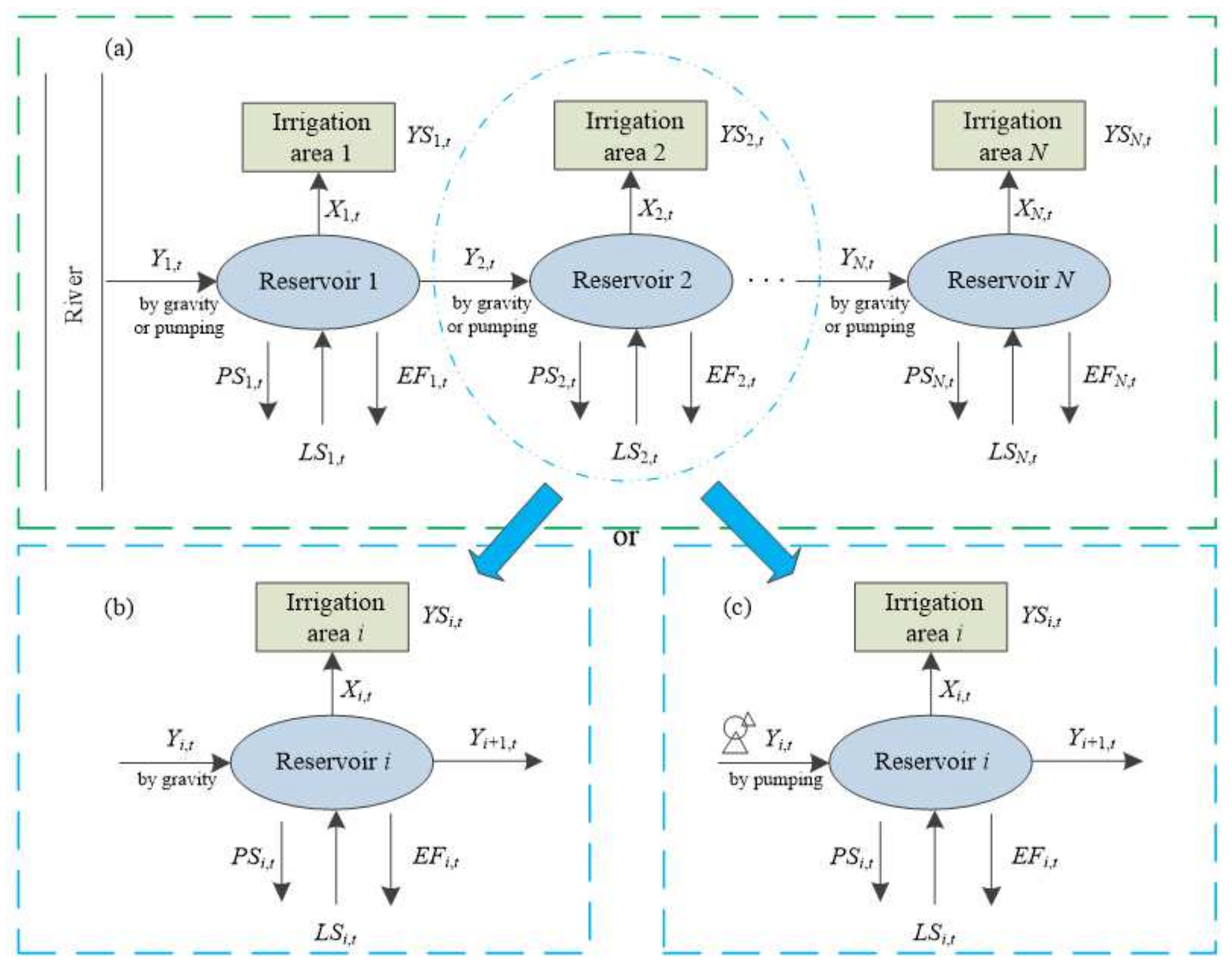

Figure 1

A system of reservoirs in series Note: (a) a system of reservoirs in series; (b) a subsystem of a reservoir; (c) a subsystem of a reservoir and a pumping station 


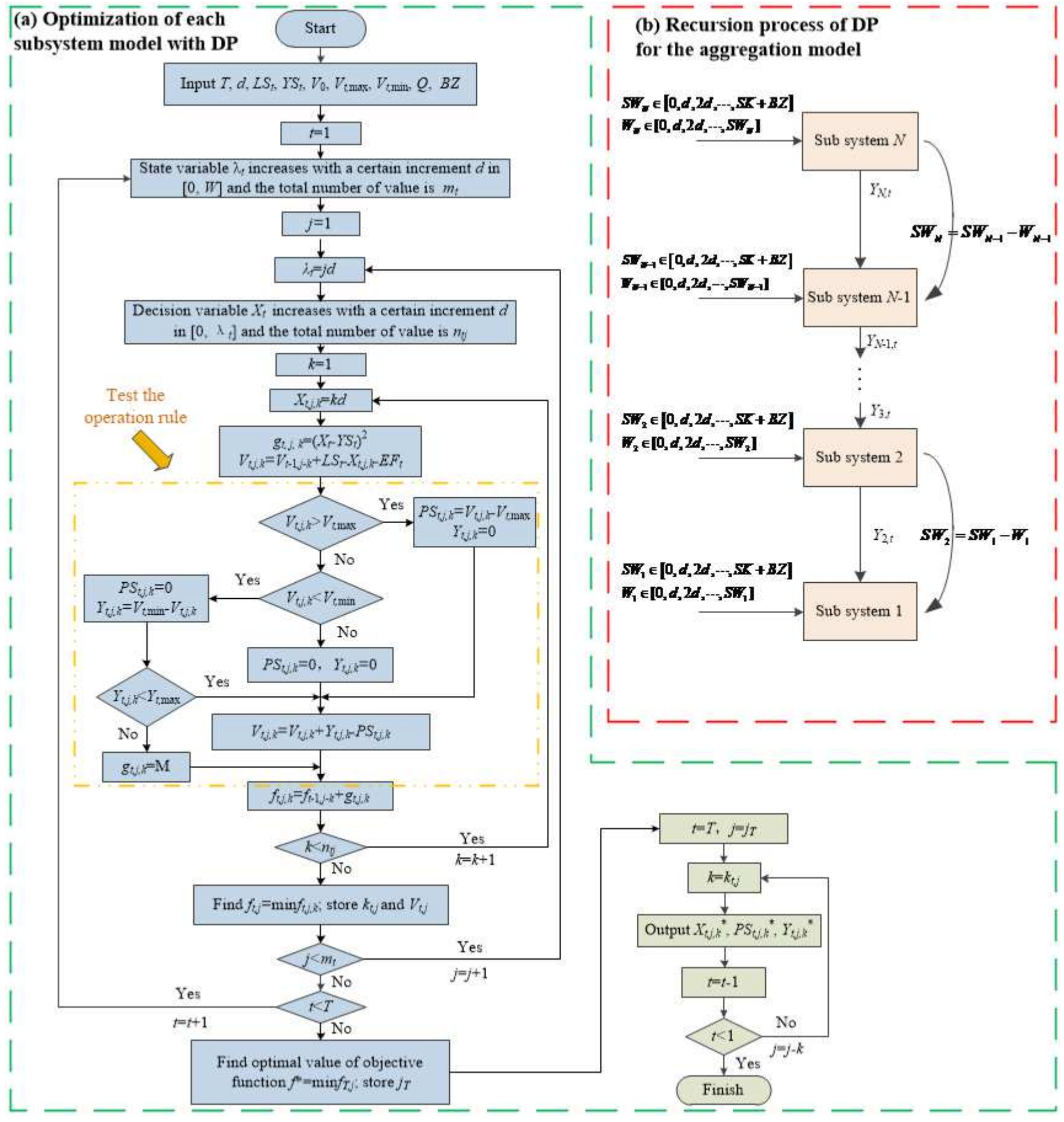

Figure 2

Flow chart of DDPA Note: $d$ is a certain increment; the aggregation model is optimized in reverse order so that $\mathrm{Yi}+1$ is a known quantity when $\mathrm{Vi}$ is calculated according to Equation (6); $\mathrm{M}$ is a positive number, which is large enough. 


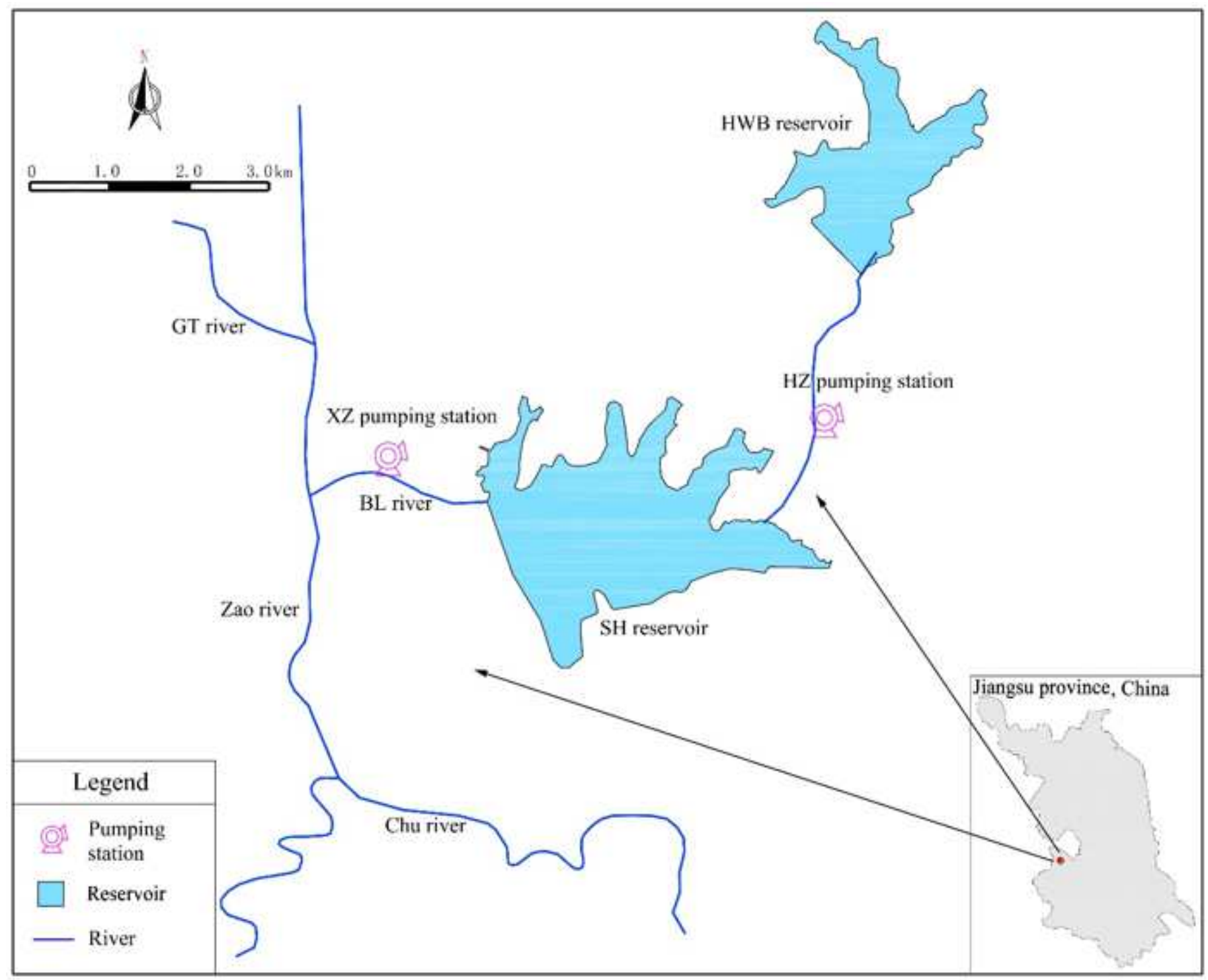

\section{Figure 3}

Location of the system Note: The designations employed and the presentation of the material on this map do not imply the expression of any opinion whatsoever on the part of Research Square concerning the legal status of any country, territory, city or area or of its authorities, or concerning the delimitation of its frontiers or boundaries. This map has been provided by the authors. 


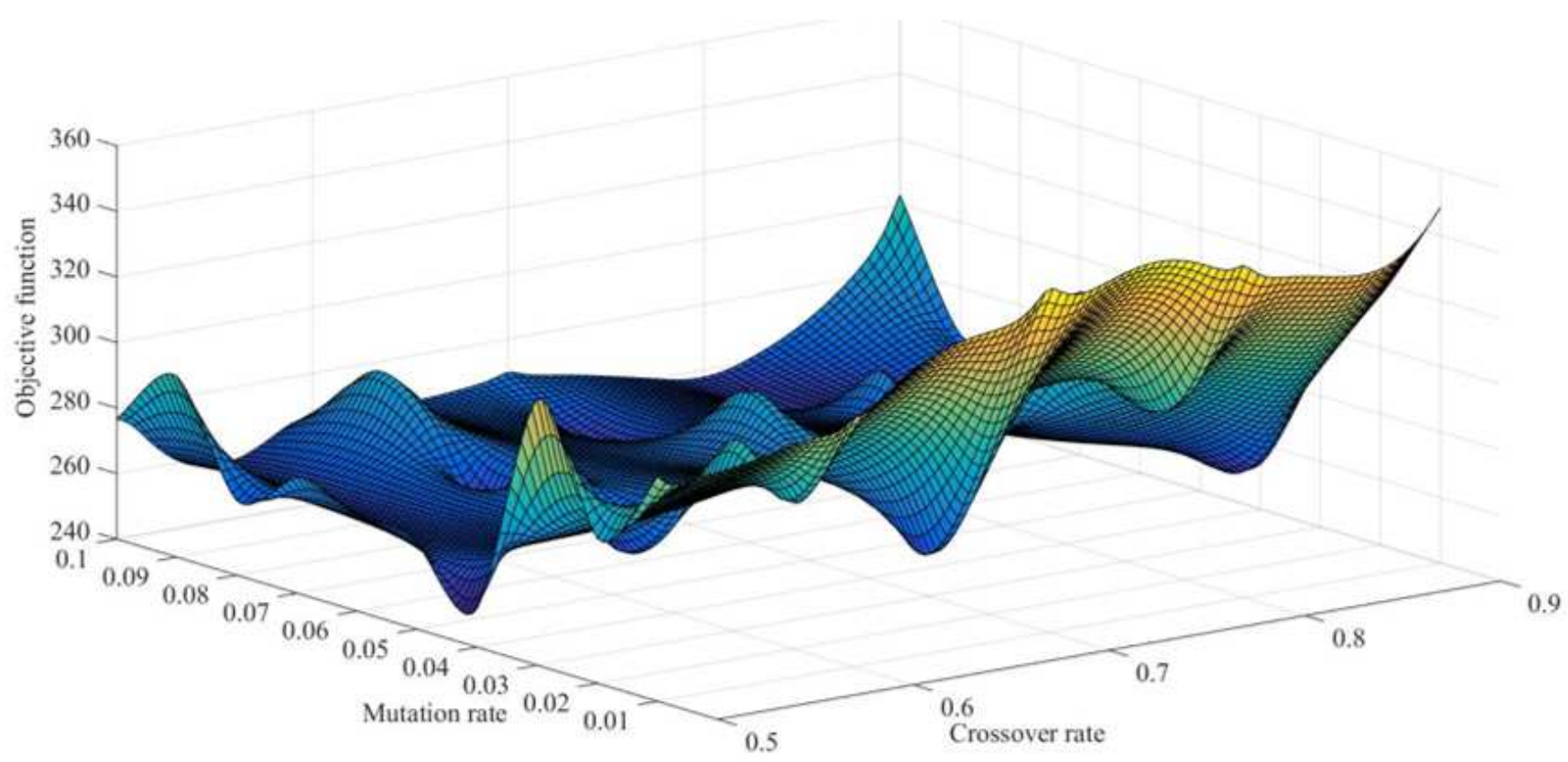

(a)

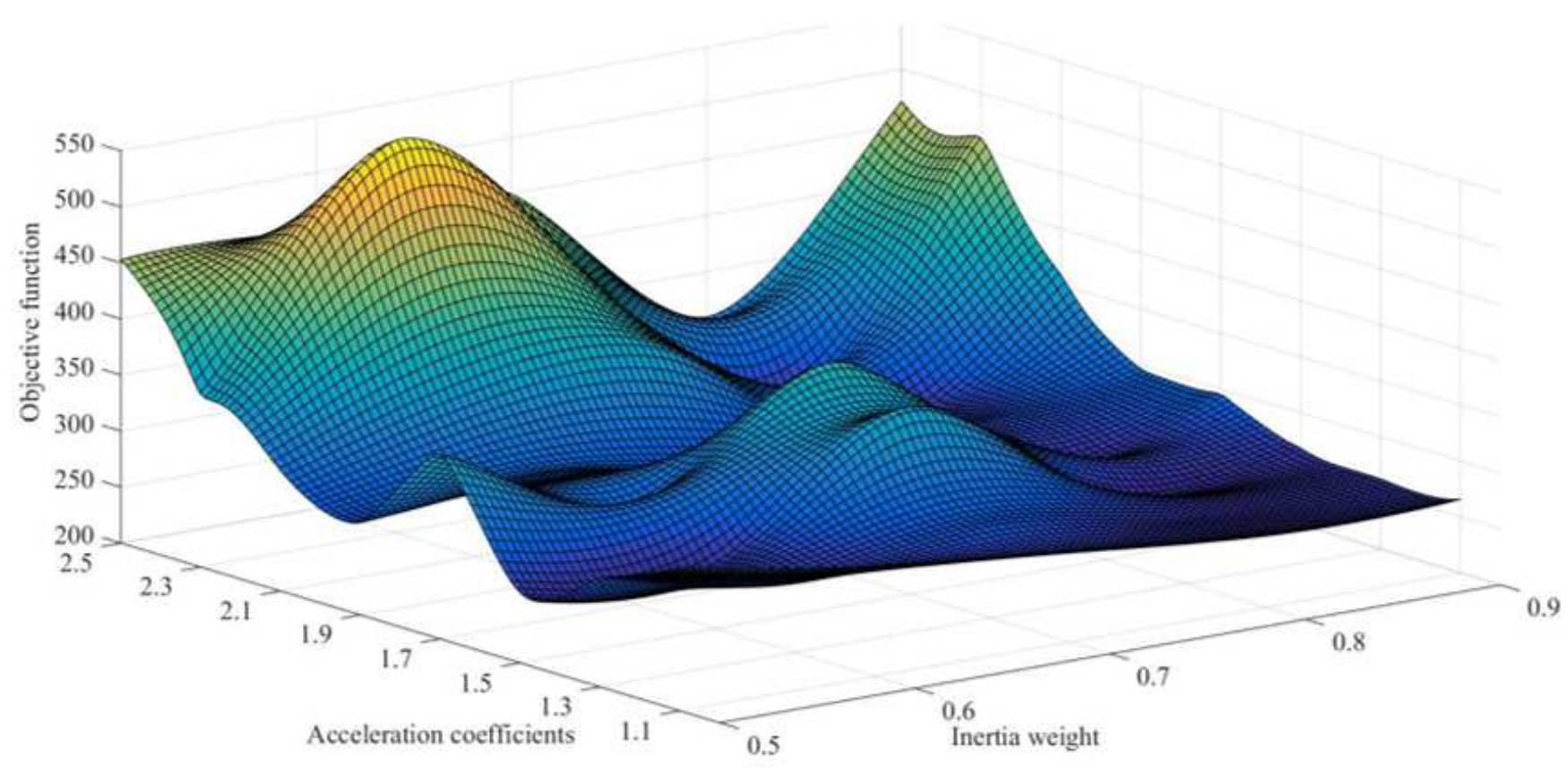

(b)

\section{Figure 4}

The objective function values obtained with GA and PSO (a) Genetic algorithm (b) Particle swarm optimization algorithm Notes: Each objective function value under different combinations of parameters is the minimum value among five runs. 